\title{
The role of telemedicine and e-health in the management of inflammatory bowel disease: improving patient outcomes
}

This article was published in the following Dove Press journal:

Smart Homecare Technology and TeleHealth

28 January 2015

Number of times this article has been viewed

\author{
Leyla J Ghazi',2 \\ Raymond K Cross' \\ 'Division of Gastroenterology and \\ Hepatology, Department of Medicine, \\ University of Maryland School of \\ Medicine, ${ }^{2}$ Veterans Affairs, Maryland \\ Heath Care System, Baltimore, MD, \\ USA
}

\begin{abstract}
Telemedicine has been used successfully in monitoring patients with a variety of chronic illnesses. Studies in the field of gastroenterology have shown that telemedicine is a feasible and well accepted method of patient monitoring. Several inflammatory bowel disease (IBD) centers have developed telemedicine systems to improve the management of these complex diseases. Implementation of these systems is feasible, and telemedicine is well received by patients. Telemedicine has been shown to improve disease activity, quality of life, adherence with short-term treatment, and to shorten relapses. Telemedicine has also been shown to decrease health care utilization; however, use of telemedicine systems is also associated with increased "non-billable" encounters (phone calls and electronic messages). Telemedicine has also been shown to be an effective mechanism to provide remote care in areas without access to IBD specialty care. It is likely that telemedicine will be increasingly utilized in the future as an adjunct to traditional monitoring, especially for patients at high risk for nonadherence, and for those with limited access to IBD centers of excellence, to improve patient self-management, and as a mechanism to provide education, health maintenance, and medication reminders to patients.
\end{abstract} Keywords: IBD, patient self-management, remote care, adherence to treatment, quality of life, health care utilization, telemedicine

\section{Introduction}

The National Digestive Diseases Information Clearinghouse has reported that 60-70 million individuals in the United States are affected by a variety of digestive diseases. ${ }^{1}$ Chronic digestive conditions such as chronic pancreatitis, cirrhosis, chronic constipation, celiac disease, inflammatory bowel disease (IBD), gastroesophageal reflux disease, irritable bowel syndrome, and viral hepatitis make up a significant proportion of these conditions. The cost burden associated with these diseases is high, with direct and indirect medical costs estimated as \$98 billion and \$44 billion, respectively. ${ }^{1}$

IBD, comprised of ulcerative colitis and Crohn's disease, are chronic inflammatory conditions of the gastrointestinal system that affect approximately 1.4 million people in the United States. ${ }^{2}$ The disease course is typically chronic, characterized by periodic exacerbations followed by symptom-free intervals. ${ }^{3}$ Symptoms including abdominal pain, diarrhea, rectal bleeding, and weight loss can have a profound and negative impact on the quality of life of patients with IBD. ${ }^{4}$

Challenges exist in the care of patients with IBD. First, in patients with active symptoms, effective therapy needs to be initiated to induce remission. Most patients respond to induction therapy with only a subset of patients having continuous, unrelenting symptoms. ${ }^{4}$ Nevertheless, despite advances in medical therapy, a significant need 
exists for novel therapies for patients with IBD. In addition, despite the excellent safety profile of most medical treatments for IBD, side effects can occur, resulting in discontinuation of drug therapy and fear of new treatments. ${ }^{5-7}$ IBD patients not responding to medical therapy often require surgery. It is estimated that $70 \%$ of patients with Crohn's disease undergo at least one resection in their lifetime, whereas $25 \%$ of patients with ulcerative colitis will undergo colectomy. ${ }^{8}$ Once remission is achieved, patients usually require chronic medical treatment to prevent recurrence of symptoms. Not surprisingly, medication adherence is challenging as symptoms are often not present to enforce medication adherence. In addition, as most patients are diagnosed at a young age, ${ }^{2}$ the diagnosis itself can have a profound negative impact on mental health as patients are forced to cope with a chronic illness while attending school, when forming intimate relationships, and when entering the work force. These factors likely contribute to a high prevalence of concurrent anxiety and depression in patients with IBD. A recent cross-sectional study from Faust et al demonstrated that approximately 50\% and $25 \%$ of patients screen positive for anxiety and depression, respectively. ${ }^{9}$

Therefore, the management of IBD patients includes not only the implementation of efficacious and safe drug therapy, but also the development of systematic patient care planning. Individualized therapy, self-management, utilization of nurse specialists in patient management, and telemedicine can be useful to achieve this goal. Providing these resources for patients with IBD can be labor intensive. A recent study from a large tertiary referral center reported that 125 calls per day are generated to support the care of patients with IBD, with an average number of calls per year of ten per patient. ${ }^{10}$

\section{Barriers to IBD care}

Effective treatments exist for IBD, but successful use is impeded by poor patient adherence. Nonadherence describes the inability of patients to follow established treatment plans, such as taking prescribed medications, attending follow-up visits and procedures, and implementing preventive health measures. In clinical practice, a significant proportion of patients are nonadherent to medical therapy, resulting in higher rates of disease flares with coinciding increased healthcare costs. Nonadherence rates for short-term therapy have been estimated between $20 \%$ and $40 \%$, but can be as high as $70 \%$ for maintenance therapies. ${ }^{11}$ Nonadherence is associated with relapses of disease as nonadherent patients are five times more likely to have a flare compared to adherent patients. ${ }^{12}$ Unintentional nonadherence, such as forgetfulness in taking medications, has been found to occur in $31 \%$ of patients. ${ }^{13}$ In a large survey study of Canadian IBD patients, cost was the most commonly reported obstacle in taking medications regularly (25\%), followed by side effects (13\%). ${ }^{14}$ Patient miscomprehension and/or misinformation is another important factor leading to nonadherence and was found in $62 \%$ of patients with ulcerative colitis. ${ }^{15}$ A new patient-provider relationship of less than 1 year is another risk factor for nonadherence. Scheduling a follow-up appointment with the medical provider and lower total discordance (defined as the difference between patients' and physicians' perceptions of health status) has been associated with lower risk of intentional nonadherence. ${ }^{13}$ The above findings suggest that improvements in provider-patient communication and in patient education might enhance adherence.

Suboptimal monitoring of IBD patients is also a potential barrier to care. It is well known that exacerbations of chronic disease are sporadic, often occurring outside of regularly scheduled office visits. When flares occur, significant delays may result before office visits are scheduled. Interestingly, Bernal et al found that a more complicated disease course characterized by steroid dependence, need for biologic agents, hospitalization, or surgery was associated with better medication adherence. ${ }^{16}$ Assuming these patients had more regular follow-up and surveillance of their disease, closer monitoring may be the reason for their better overall adherence and may increase the chance that relapses will coincide with office visits.

Other barriers to care include young age at diagnosis, potential for medication side effects, and the long-term need for treatment, all of which can contribute to decreased adherence and subsequent risk for worsening of the disease. ${ }^{11}$ Cross et al found that approximately $70 \%$ of outpatients with IBD in a tertiary referral center reported medication side effects; more than half of these were considered moderate to severe by patients. Fear of adverse events related to medications has a negative impact on adherence. ${ }^{14,17}$ Polypharmacy is another potential problem in patients with chronic illness such as IBD. Cross et al have reported that $50 \%$ of patients in a tertiary referral center use five or more medications; these patients had significantly higher disease activity and decreased quality of life compared to those on fewer medications. Managing side effects of medical therapy in patients with IBD is a complex undertaking. The task of reviewing medications lists at each clinical visit can be daunting and time-consuming. Ongoing education is essential to help patients differentiate side effects from intensifying disease activity. 
A number of other barriers contribute to suboptimal outcomes in patients with IBD. Patient knowledge about the disease is quite limited, and concerted efforts to improve patients' knowledge have been ineffective. ${ }^{18}$ Access to care is another barrier to improved treatment outcomes. As IBD care has become increasingly complicated, patients and providers often seek consultation with an IBD specialist. Most urban areas have gastroenterologists and surgeons with an interest in care of this population. However, rural areas often have limited access to IBD care and even in urban areas, some patients have limited resources to be evaluated in areas of IBD expertise (Table 1).

Self-management programs have been suggested as an effective means for long-term treatment and follow-up in patients with IBD. Self-management is used in other chronic diseases such as diabetes and asthma, and could be used to more rapidly treat flares and to improve patient confidence to complete tasks ("self-efficacy") in IBD. A randomized controlled trial by Robinson et al found that use of a selfmanagement program in patients with ulcerative colitis after discharge from the hospital facilitated early treatment of relapses and reduced routine follow-up visits. ${ }^{19}$ Using a similar system, Kennedy et al found that self-management resulted in fewer hospitalizations and improved coping by patients..$^{20}$ Together, these two studies demonstrate that providers can offer written action plans for patients for management of acute symptoms resulting in early initiation of treatment, decreased office visits, and hospitalizations, and improved patient coping.

\section{Description of telemedicine systems for IBD}

Telemanagement is a telemedicine system designed to assist providers in monitoring patients, to provide patient education, and to assist patients in following

Table I Barriers to successful outcomes in inflammatory bowel disease care

I. Nonadherence to
a. Medications
b. Clinical follow-up
c. Scheduling of diagnostic tests
d. Preventative screening
2. Patient miscomprehension or misinformation regarding
a. Medication side effects
b. Disease course, prognosis, and symptoms
3. Limited access to inflammatory bowel disease specialized care
4. Limited resources to provide coordinated care and education for
patients
5. Ineffective treatments available

self-care plans. The home automated telemanagement system (HAT) was originally developed for patients with asthma and other chronic respiratory diseases. ${ }^{21}$ HAT was modified at the University of Maryland, Baltimore Inflammatory Bowel Disease Program for use in patients with IBD (IBD HAT). IBD HAT is comprised of three components: a home unit (laptop computer linked to a weight scale), a decision support system, and a web portal for providers.

As depicted in Figure 1, the home unit includes an electronic scale connected to a refurbished laptop via a serial port. Using the laptop, patients respond to a series of questions regarding IBD symptoms, side effects of medications, and doses of medications taken since the last testing session. Body weight is measured after completion of all patient reported outcome measures. Alerts are triggered based on the responses to each question and to changes in body weight. Alerts are based on responses to all questions as well as responses to core symptoms including overall well-being, restriction in social activities, diarrhea, and abdominal pain. Patients also receive an IBD-related educational prompt once per week in the format of a "tip of the day", which they are tested on the following week. Patients must answer questions correctly in order to advance in the educational curriculum. Patients can communicate with providers through an electronic messaging system.

The IBD HAT secure website provides an interface for the collected patient data. The care coordination team including physicians, nurse coordinators, and research staff use this portal to create patient profiles. Each profile contains information regarding disease history, medications, and testing schedules, which is synchronized with the home unit. Alerts are generated and sent to providers if

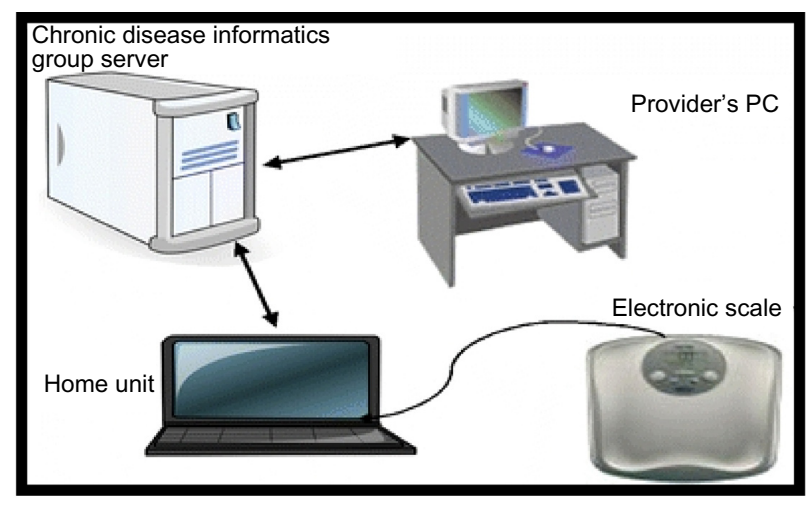

Figure I Model of the home telemanagement (IBD HAT) system for inflammatory bowel disease.

Abbreviations: IBD HAT, inflammatory bowel disease home automated telemanagement system; PC, personal computer. 
certain individualized criteria are exceeded (for example, if number of bowel movements exceed expected threshold). Tabulated and graphical forms of the test data and trends can be reviewed after self-testing.

The IBD HAT system was subsequently modified for ulcerative colitis (UC HAT) to incorporate self-management plans or action plans and to allow for use of a cellular phone to transmit results instead of a "land line". Providers develop specific action plans for each patients based on the severity of symptoms, which are delivered after each self-testing session. Depending upon scores generated after each session, participants receive action plans in one of three categories: 1) green zone, if no to mild symptoms; 2) yellow zone, if moderate symptoms; or 3) red zone, if severe symptoms. Based on the assessment zone, providers can choose one or more actions from a list of options which can be modified over time as needed. Telemedicine for patients with IBD (TELE-IBD) was a third iteration of the telemanagement system that replaced home testing units with use of cellular phones. In this system, questions about symptoms, weight, side effects, medication prompts, and educational messages are sent via text message and immediately available for review on a secure website. Zone assessment, action plans, and messages from the provider and research staff is sent via text immediately after selftesting or as needed.

Constant Care is another telemedicine system developed in Denmark. Construction of the 24-hour Constant Care website began in 2001, and is available in both Danish and English. ${ }^{22}$ Participants undergo a 1.5-hour training session on a variety of topics including but not limited to the etiology, pathology, and anatomy of IBD, medical and surgical treatments, adherence, prevention, nutrition, women's health, and use of the http://www.constant-care.dk website. Clinic visits were scheduled for each group, but the web-based patients could cancel appointments if they felt well. After training, the web-based patients complete self-assessments via the Constant Care website once per month until they experience a relapse, defined as rectal bleeding, three or more bowel movements daily, or nighttime bowel movements. In case of relapse, patients log on daily to complete an assessment of disease activity and to initiate a short course of treatment with oral or topical aminosalicylate and/or oral or topical steroids. Participants continue self-assessment until they enter the green zone. Patients then complete assessments once a week for a total of 4 weeks; patients then resume routine self-assessment monthly. Symptoms are categorized in a "traffic light" manner similar to UC HAT.

\section{The role of telemedicine in improving patient outcomes: an evidence-based approach}

Telemedicine has been used successfully in chronic conditions such as asthma, diabetes, and congestive heart failure. In asthma, telemedicine has been shown to improve symptoms, quality of life, and adherence. It has decreased the use of quick relief inhalers and urgent care visits. ${ }^{23,24}$ In patients with diabetes, telemedicine improves glycemic control and glycosylated hemoglobin levels. ${ }^{25-27}$ Telemedicine improves quality of life, and decreases hospitalizations and other health care costs in congestive heart failure. ${ }^{28,29}$ These chronic conditions share similarities with IBD in that they are chronic, characterized by periods of disease worsening, and require continuous treatment with medications to control symptoms and/or maintain health.

Telemedicine has been shown to be an effective tool within gastroenterology, namely in treatment of patients with chronic hepatitis $\mathrm{C}$ and irritable bowel syndrome. Telemedicine has been used in hepatitis $\mathrm{C}$ to provide guidance and training of primary care providers by hepatologists in use of anti-viral therapies to patients in settings where hepatologists are unavailable. Prior studies have shown that patients with irritable bowel syndrome can complete a webbased questionnaire and receive cognitive behavioral therapy using telemedicine systems. ${ }^{30-33}$

Cross et al assessed the acceptance of IBD HAT in a pilot study of ten patients with Crohn's and colitis. ${ }^{34}$ Mean duration of disease was 15 years. Thirty percent of patients reported limited disease knowledge, one had never used the Internet, and two did not use a home computer. However, patients found that self-test procedures were uncomplicated, easy to use, and took little time. Importantly, $30 \%$ of patients reported that they would feel significantly or moderately safer while using this system. All participants felt that weekly testing was realistic.

The acceptance and feasibility of the IBD HAT system was confirmed in a subsequent prospective study among 34 patients. ${ }^{35}$ Participants completed weekly self-testing for 6 months. Overall, adherence to self-testing was 91\%. Similar to the prior study, IBD HAT was found to have ease of use and not interfere with daily activities. In addition, participants' satisfaction with treatment and disease-state knowledge improved significantly after using IBD HAT. 
There were trends toward improved disease activity and quality of life after use of telemanagement for 6 months. ${ }^{35} \mathrm{~A}$ small follow-up study $(n=10)$ of a telemanagement system specific for patients with UC (UC HAT) confirmed that patient acceptance and feasibility of telemedicine was high. ${ }^{36}$

Subsequently, 47 ulcerative colitis patients were randomized to UC HAT or best available care. ${ }^{37}$ Participants in the telemanagement group completed self-testing weekly. At baseline, participants in the best available care group had higher disease-specific quality of life scores and were significantly less likely to use immune suppressants compared to the UC HAT group. A greater number of participants (eight) withdrew in the intervention arm versus one withdrawal in the best available care group. After adjusting for baseline quality of life scores, disease activity scores decreased 12 points from baseline in the UC HAT arm $(P=0.08)$ compared to one point in the best available care arm $(P=0.84)$. After adjustment for baseline knowledge, UC HAT participants experienced a 16-point improvement in quality of life scores from baseline compared to the best available care group $(P=0.04)$. Self-reported adherence was not significantly changed in either arm compared to baseline. ${ }^{37}$

Elkjaer et al reported results on the feasibility and acceptance of a telemanagement program for ulcerative colitis patients and described the development and validation of the Constant Care website. ${ }^{38}$ Ten participants with UC and five of their relatives participated in the validation study of the Constant Care website. Patients were being treated with conventional medical therapy including aminosalicylates, azathioprine, or infliximab. The majority of participants expected to see an improvement in quality of life, quality of treatment, and knowledge of disease with use of the Constant Care website after the training session.

A randomized controlled trial in Denmark and Ireland was conducted to assess the effectiveness of the Constant Care web system versus conventional care in ulcerative colitis. ${ }^{38}$ Of the 333 participants from both countries, only 135 participants completed the 1-year follow-up visit. The control group was more likely to be female $(70 \%$ vs $51 \%$ in the web-based group), and older (median 48 vs 41 years of age in the web-based group). In the Danish arm, 5-aminosalicylate adherence measured by medication refills was not different between the web and control groups. However, the web group experienced significantly higher adherence to acute treatment. IBD knowledge, quality of life, general health, vitality, and emotional and social functioning was higher in the web group compared to controls. Although approximately half of all participants experienced a flare of symptoms during the study, there was no difference in flare rates between the web group and controls. However, relapses were significantly shorter in the web group compared to controls (18 days vs 77 days, respectively; $P<0.0001){ }^{38}$

Similarly, in the Irish arm, medication adherence to acute treatment was significantly greater in the web group, as was mental health, physical functioning, and social functioning as compared to controls. ${ }^{38}$ There were no differences between the groups in terms of IBD knowledge. The web group experienced more relapses than controls; however, these were shorter than the relapses experienced by controls (30 days vs 70 days, respectively; $P<0.03$ ). Controls needed more ulcerative colitis-related acute visits compared to the web group (21 vs 107 visits, respectively; $P<0.0001$ ). However, web participants sent more emails $(n=86)$ and called the office more frequently $(n=21)$ than controls (seven emails and 17 phone calls). Fecal calprotectin levels were elevated in a higher percentage of controls than web participants. ${ }^{38}$

Overall, $88 \%$ of web group participants preferred telemedicine to conventional care. ${ }^{38}$ Web participants felt safer and empowered by the ability to initiate treatment using the Constant Care website for structured guidance. However, high lost-to-follow-up rates were seen in this study, similar to a prior prospective study. ${ }^{37}$

In a follow-up, open-label, prospective study, 95 patients with mild-to-moderate ulcerative colitis utilized web-guided therapy with mesalamine (http://www.meza.constantcare.dk). Patients completed disease activity assessments and electronic assessment of fecal calprotectin weekly. Adherence over 3 months was higher in this study, with $86 \%$ of patients adherent to web-guided therapy. Importantly, patients experienced significant improvements in disease activity as measured by disease activity indices and by lower levels of calprotectin. The investigators developed an inflammatory burden score based on the combination of symptom self-report and calprotectin levels; using this composite index, the mesalamine dose was de-escalated in nearly $90 \%$ of patients from $4.8 \mathrm{~g}$ per day at baseline to $2.4 \mathrm{~g}$ per day at week 12 . Pederson et al has also evaluated the utility of Constant Care in individualization of infliximab therapy in patients with Crohn's disease. ${ }^{39}$ Inflammatory burden was calculated using a combination of weekly selfreported symptoms and measurement of fecal calprotectin. Patients with moderate to severe inflammatory burden were assessed as being in the yellow or red zone. These patients 
were instructed to schedule an infliximab infusion. Using this strategy, $50 \%$ of patients were able to receive infliximab at a longer interval ( $>8$ weeks) without adverse outcomes. ${ }^{39}$

A multicenter, randomized, controlled trial(NCT01692743) is ongoing to determine whether TELE-IBD improves disease activity, quality of life, and health care utilization. IBD patients with a flare within 2 years of enrollment are eligible to receive weekly monitoring, every-other-weekly monitoring, or standard care. As of September 2014, 240 participants have been enrolled, with 22 completing 1-year of follow-up. Importantly, only eleven participants have withdrawn from the study, a much better attrition rate than previously described, ${ }^{37,38}$ suggesting that improved delivery of telemedicine and increasing patient acceptance of the technology improves adherence with self-testing.

The feasibility of telemedicine (teleconsultation) has also been evaluated in a Veterans Affairs population. A prospective, randomized, controlled, pilot study was performed to evaluate the effectiveness and applicability of Collaborative Imaging. ${ }^{40}$ Veterans were randomized to have a teleconsultation with an IBD provider or to have a conventional in-person encounter with a gastroenterologist. Patients and specialist providers utilized two standard Apple computers to establish communication between the Palo Alto Veterans Affairs and the San Fransisco Veterans Affairs. Thirty-four Veterans with Crohn's disease and ulcerative colitis underwent 57 encounters over 9 months. Participants in both groups rated their clinic experience, clinical satisfaction with provider attention to concerns, bedside manner, and skill level of the doctor highly. Importantly, the number of Veterans seen per clinic session and duration for clinic visits were similar between the two groups. In addition, each clinic session lasted approximately 1 hour in both groups. ${ }^{40}$

\section{Prospects for the management of IBD care through telemedicine and e-health}

Telemedicine is universally well accepted by patients with IBD. Longitudinal use of these systems seems feasible and is associated with improved clinical outcomes. Prospective

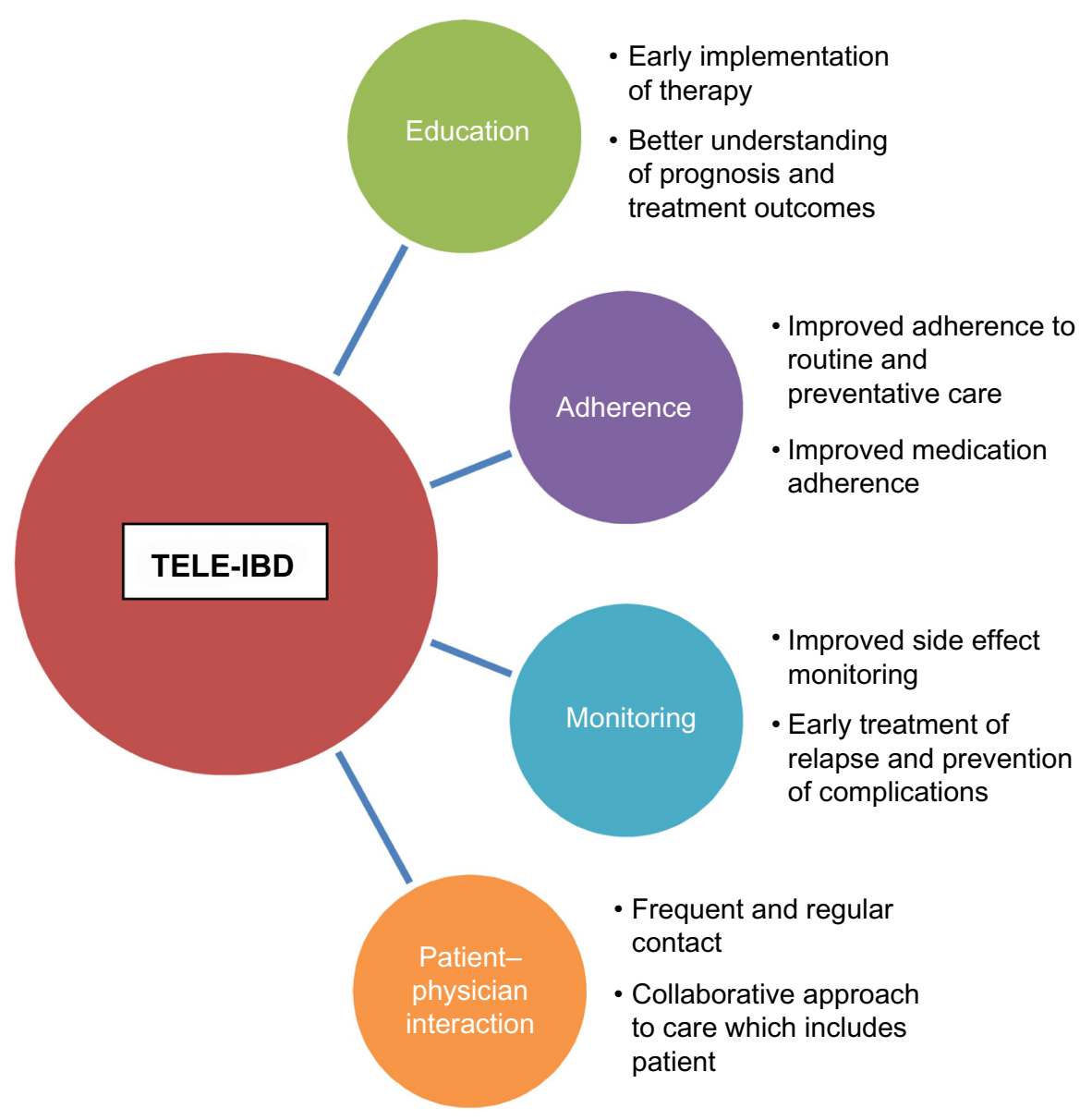

Figure 2 The use of telemanagement in improving IBD care.

Abbreviations: IBD, inflammatory bowel disease; TELE-IBD, telemedicine for patients with IBD. 
studies $^{37,38}$ have shown that use of telemedicine is associated with improved disease activity, disease-specific and general quality of life, adherence to treatment of acute flares, and improved knowledge. In addition, surrogate markers of disease activity such as fecal calprotectin levels have also improved after use of telemedicine systems. Despite these improvements, the largest study ${ }^{38}$ to date from Europe reported higher rates of flares in patients monitored with telemedicine; however, the duration of flares was significantly less compared to controls. Health care utilization was less in patients monitored with telemedicine, but non-billable services such as telephone calls and electronic messages were increased. Despite the positive effects seen in trials ${ }^{35,37-39,41}$ thus far, the high attrition raises concerns about long-term adherence to telemonitoring systems. We hypothesized that use of a portable device like a cellular phone would improve adherence. This is supported by the high rates of retention reported in our ongoing multicenter trial. ${ }^{38}$ It is also possible that over time, patients are increasingly accepting of technology like telemedicine, thereby improving adherence. We await the full results of our trial to determine the impact of telemedicine on disease activity, quality of life, and health care utilization in a large, referral population from three academic medical centers (University of Maryland, Baltimore, Maryland, USA; University of Pittsburgh Medical Center, Pittsburgh, PA, USA; and Vanderbilt University, Nashville, TN, USA).

\section{Conclusion}

Although significant advances continue in the treatment of IBD, major problems still exist in the delivery of health care. This suggests that factors other than improved medical therapy are important determinants of successful patient outcomes. These include nonadherence, adverse drug reactions, suboptimal monitoring, poor patient education, and physician-patient discordance. In our changing health care environment, physicians and patients have increasing demands on their time and with the explosion of information technology, emerging techniques such as telemedicine are needed to improve care. If telemedicine results in improved adherence, improved monitoring of symptoms and medications, earlier implementation of medical therapy, more frequent patient-provider interactions, and improved patient self-management, then decreases in clinical disease activity and improvements in quality of life are likely (Figure 2 ). Better control of IBD will result in decreased urgent care visits and hospitalizations, shorter lengths of hospital stays, and decreased health care costs. If telemedicine improves outcomes as expected and can be implemented at low cost, it can improve the quality, safety, efficiency, and effectiveness of health care for individuals with IBD. Furthermore, telemedicine could be used in high-risk subgroups of IBD patients such as adolescents, patients with limited access to medical care, patients initiating novel drug therapy during a flare of disease, and patients with risk factors for nonadherence. Moreover, telemedicine can be used to provide IBD care in areas where access to gastroenterologists, let alone IBD specialists, is limited. Lastly, telemedicine could be utilized by IBD practices to improve quality of care delivered to patients with IBD.

\section{Disclosure}

Raymond $\mathrm{K}$ Cross, MD, MS, has received education and research grants from Abbvie, Janssen, Shire, and Takeda. He has received income for consulting from Abbvie and Janssen, and has participated in advisory boards for Abbvie, Janssen, and Takeda. Leyla J Ghazi, MD, has participated in advisory boards for Abbvie and Continuing Medical Education consulting program for UCB, Inc. The authors report no other conflicts of interest in this work.

\section{References}

1. US Department of Health and Human Services. NIH (National Institute of Diabetes and Digestive and Kidney Diseases). Webpage on Internet. Digestive Diseases A-Z. Available from: http://www.niddk.nih.gov/ health-information/health-topics/digestive-diseases/Pages/default.aspx. Accessed December 8, 2014.

2. Loftus CG, Loftus EV Jr, Harmsen WS, et al. Update on the incidence and prevalence of Crohn's disease and ulcerative colitis in Olmsted County, Minnesota, 1940-2000. Inflamm Bowel Dis. 2007;13: 254-261.

3. Faubion WA Jr, Loftus EV Jr, Harmsen WS, ZinsmeisterAR, Sandborn WJ. The natural history of corticosteroid therapy for inflammatory bowel disease: a population-based study. Gastroenterology. 2001;121(2): $255-260$.

4. Loftus EV Jr, Schoenfeld P, Sandborn WJ. The epidemiology and natural history of Crohn's disease in population-based patient cohorts from North America: a systematic review. Aliment Pharmacol Ther. 2002;16(1):51-60.

5. Bajaj JS, Saeian K, Varma RR, et al. Increased rates of early adverse reaction to azathioprine in patients with Crohn's disease compared to autoimmune hepatitis: a tertiary referral center experience. $A m ~ J$ Gastroenterol. 2005;100(5):1121-1125.

6. Cross RK, Wilson KT, Binion DG. Polypharmacy and Crohn's disease Aliment Pharmacol Ther. 2005;21(10):1211-1216.

7. Cross RK, Lapshin O, Finkelstein J. Patient subjective assessment of drug side effects in inflammatory bowel disease. J Clin Gastroenterol. 2008;42(3):244-251.

8. Munkholm P, Langholz E, Davidsen M, Binder V. Intestinal cancer risk and mortality in patients with Crohn's disease. Gastroenterology. 1993;105(6):1716-1723.

9. Faust AH, Halpern LF, Danoff-Burg S, Cross RK. Psychosocial factors contributing to inflammatory bowel disease activity and health-related quality of life. Gastroenterol Hepatol (N Y). 2012;8(3):173-181.

10. Ramos-Rivers C, Regueiro M, Vargas EJ, et al. Association between telephone activity and features of patients with inflammatory bowel disease. Clin Gastroenterol Hepatol. 2014;12(6):986-994. 
11. Kane SV, Cohen RD, Aikens JE, Hanauer SB. Prevalence of nonadherence with maintenance mesalamine in quiescent ulcerative colitis. Am J Gastroenterol. 2001;96(10):2929-2933.

12. Kane S, Huo D, Aikens J, Hanauer S. Medication nonadherence and the outcomes of patients with quiescent ulcerative colitis. Am J Med. 2003;114(1):39-43.

13. Sewitch MJ, Abrahamowicz M, Barkun A, et al. Patient nonadherence to medication in inflammatory bowel disease. Am J Gastroenterol. 2003; 98(7):1535-1544.

14. Ediger JP, Walker JR, Graff L, et al. Predictors of medication adherence in inflammatory bowel disease. Am J Gastroenterol. 2007;102(7): 1417-1426.

15. Martin A, Leone L, Castagliuolo I, Di Mario F, Naccarato R. What do patients want to know about their inflammatory bowel disease? Ital J Gastroenterol. 1992;24(9):477-480.

16. Bernal I, Domènech E, Garcia-Planella E, et al. Medication-taking behavior in a cohort of patients with inflammatory bowel disease. Dig Dis Sci. 2006;51(12):2165-2169.

17. Kane SV. Strategies to improve adherence and outcomes in patients with ulcerative colitis. Drugs. 2008;68(18):2601-2609.

18. Quan H, Present JW, Sutherland LR. Evaluation of educational programs in inflammatory bowel disease. Inflamm Bowel Dis. 2003;9(6): 356-362.

19. Robinson A, Thompson DG, Wilkin D, Roberts C; Northwest Gastrointestinal Research Group. Guided self-management and patientdirected follow-up of ulcerative colitis: a randomised trial. Lancet. 2001;358(9286):976-981.

20. Kennedy AP, Nelson E, Reeves D, et al. A randomised controlled trial to assess the effectiveness and cost of a patient orientated self management approach to chronic inflammatory bowel disease. Gut. 2004;53(11): 1639-1645

21. Finkelstein J, O'Connor G, Friedmann RH. Development and implementation of the home asthma telemonitoring (HAT) system to facilitate asthma self-care. Stud Health Technol Inform. 2001; 84(Pt 1):810-814.

22. Elkjaer M, Burisch J, Avnstrøm S, Lynge E, Munkholm P. Development of a Web-based concept for patients with ulcerative colitis and 5-aminosalicylic acid treatment. Eur J Gastroenterol Hepatol. 2010; 22(6):695-704.

23. Joshi A, Amelung P, Arora, M, Finkelstein J. Clinical Impact of home automated telemanagement in asthma. AMIA Annu Symp Proc. 2005; 1000 .

24. Finkelstein J, Hripcsak G, Cabrera M. Telematic system for monitoring of asthma severity in patients' homes. Stud Health Technol Inform. 1998;58(Pt 1):272-276.

25. Shea S, Weinstock RS, Starren J, et al. A randomized trial comparing telemedicine case management with usual care in older, ethnically diverse, medically underserved patients with diabetes mellitus. $\mathrm{J} \mathrm{Am}$ Med Inform Assoc. 2006;13(1):40-51

26. Montori VM, Helgemoe PK, Guyatt GH, et al. Telecare for patients with type 1 diabetes and inadequate glycemic control: a randomized controlled trial and meta-analysis. Diabetes Care. 2004;27(5):1088-1094.
27. Dang S, Ma F, Nedd N, Florez H, Aguilar E, Roos BA. Care coordination and telemedicine improves glycaemic control in ethnically diverse veterans with diabetes. J Telemed Telecare. 2007;13(5):263-267.

28. Roth A, Kajiloti I, Elkayam I, Sander J, Kehati M, Golovner M. Telecardiology for patients with chronic heart failure: the 'SHL' experience in Israel. Int J Cardiol. 2004;97(1):49-55.

29. Schofield RS, Kline SE, Schmalfuss CM, et al. Early outcomes of a care coordination-enhanced telehome care program for elderly veterans with chronic heart failure. Telemed J E Health. 2005;11(1):20-27.

30. Quezada, S, Cross RK. Telemedicine for Chronic Digestive Diseases: A Systematic Qualitative Review, Telemedicine Techniques and Applications. Rijeka, Croatia: InTech, 2011:347-362.

31. Rossaro L, Tran T, Cole S, Nesbitt T. Telemedicine: Improving Access to Care of Hepatitis C - Guest Editorial. Practical Gastroenterology. April 2003; (27):17-22.

32. Arora S, Thornton K, Jenkusky SM, Parish B, Scaletti JV. Project ECHO: linking university specialists with rural and prison-based clinicians to improve care for people with chronic hepatitis C in New Mexico. Public Health Rep. 2007. 122 Suppl 2: 74-77.

33. Arora S, Thornton K, Murata G, et al. Outcomes of Treatment for Hepatitis C Virus Infection by Primary Care Providers. New England Journal of Medicine. June 2011. Available from: http://www.nejm.org/ doi/full/10.1056/NEJMoa1009370” \t “_blank.

34. Cross RK, Arora M, Finkelstein J. Acceptance of telemanagement is high in patients with inflammatory bowel disease. J Clin Gastroenterol. 2006;40(3):200-208.

35. Cross RK, Finkelstein J. Feasibility and acceptance of a home telemanagement system in patients with inflammatory bowel disease: a 6-month pilot study. Dig Dis Sci. 2007;52(2):357-364.

36. Cross RK, Cheevers N, Finkelstein J. Home telemanagement for patients with ulcerative colitis (UC HAT). Dig Dis Sci. 2009;54(11): 2463-2472.

37. Cross RK, Cheevers N, Rustgi A, Langenberg P, Finkelstein J. Randomized, controlled trial of home telemanagement in patients with ulcerative colitis (UC HAT). Inflamm Bowel Dis. 2012;18(6): 1018-1025.

38. Elkjaer M, Shuhaibar M, Burisch J, et al. E-health empowers patients with ulcerative colitis: a randomised controlled trial of the web-guided 'Constant-care' approach. Gut. 2010;59(12):1652-1661.

39. Pedersen N, Elkjaer M, Duricova D, et al. eHealth: individualisation of infliximab treatment and disease course via a self-managed web-based solution in Crohn's disease. Aliment Pharmacol Ther. 2012;36(9): 840-849.

40. Krier M, Kaltenbach T, McQuaid K, Soetikno R. Potential use of telemedicine to provide outpatient care for inflammatory bowel disease. Am J Gastroenterol. 2011;106(12):2063-2067.

41. Pedersen N, Thielsen P, Martinsen L, et al. eHealth: Individualization of Mesalazine Treatment Through a Self-managed Web-based Solution in Mild-to-moderate Ulcerative Colitis. Inflamm Bowel Dis. 2014;20(12):2276-2285.
Smart Homecare Technology and TeleHealth

\section{Publish your work in this journal}

Smart Homecare Technology and TeleHealth is an international, peer-reviewed, open access online journal publishing original research, reviews, editorials and commentaries on the application of technology to support people and patients at home and in assisted living centers to optimize healthcare and management resources. Specific topics in the journal include: Development and application of

\section{Dovepress}

devices within the home and embedded in appliances; Healthcare provider communication and education tools; and drug ordering and adherence. The manuscript management system is completely online and includes a very quick and fair peer-review system, which is all easy to use. Visit http://www.dovepress.com/ testimonials.php to read real quotes from published authors. 